\title{
SLCO4AI is a Prognosis-Associated Biomarker Involved in Neutrophil-Mediated Immunity in Thyroid Cancer
}

\author{
Xin-Sheng Wang \\ Shi-Le Wu \\ Zhe Peng \\ Hai-Hong Zhu
}

Department of General Surgery, Qinghai Provincial People's Hospital, Xining, Qinghai, People's Republic of China
Correspondence: Xin-Sheng Wang; Hai-Hong Zhu

Department of General Surgery, Qinghai Provincial People's Hospital, 2 Gonghe Road, Chengdong District, Xining, 810007, Qinghai, People's Republic of China Tel + I I I 9765530; + I 3099775037 Email wangxinsheng1986@163.com; zhuhaihong1214@I26.com
Objective: The study aimed to investigate the value of solute carrier organic anion transporter family member 4A1 (SLCO4A1) in thyroid cancer mainly from three aspects: expression, prognosis, and biological function analyses.

Methods: Based on various bioinformatic approaches, genes co-expressed with vascular endothelial growth factor C (VEGFC) in thyroid cancer were used for further survival and expression analyses to identify the target gene. After evaluation of the SLCO4A1 expression levels in thyroid cancer, Cox regression analysis was utilized to predict the risk factors for survival of thyroid cancer patients. And receiving operating characteristic curve analysis was performed to validate the prognostic value of SLCO4A1. Additionally, WebGestalt was employed for enrichment analysis of SLCO4A1 and its co-expressed genes. Further, the relation between SLCO4A1 and neutrophil was analyzed, followed by exploring the association of SLCO4A1 with immunomodulators.

Results: A total of 38 consistent VEGFC co-expressed genes were generated, and SLCO4A1 was selected as the target gene due to its oncogenic characteristics. SLCO4A1 was highly expressed in thyroid cancer at both gene and protein levels, and SLCO4A1 mRNA expression was significantly associated with the cancer stage (all $P<0.05$ ). Besides, high SLCO4A1 expression led to unfavorable progression-free survival (PFS) of thyroid cancer patients $(P=0.0066)$. Further, Cox regression analysis indicated that high SLCO4A1 expression was an independent predictor of poor PFS in patients with papillary thyroid cancer, particularly in patients at stage 1 and female patients (all $P<0.001$ ). The enrichment analysis results showed that SLCO41A was involved in the neutrophil-mediated immunity pathway. Moreover, SLCO4A1 had a positive relation with neutrophils (all $P<0.05$ ). Finally, a significant correlation between SLCO4A1 and immunomodulators was observed (all $P<0.001)$.

Conclusion: SLCO4A1 was a potential prognostic biomarker for papillary thyroid cancer patients. And SLCO4A1 might affect PFS in thyroid cancer patients by positive regulation of neutrophil-mediated immunity pathway.

Keywords: VEGFC, SLCO4A1, thyroid cancer, bioinformatics

\section{Introduction}

Thyroid cancer belongs to one of the most frequent endocrine malignancies worldwide with a rapidly increasing incidence. ${ }^{1}$ According to statistics, more than 62,000 cases of thyroid cancer were newly diagnosed in $2015 .^{2}$ A previous study also reported that thyroid cancer cases have been increasing at a rate of $4 \%$ every year, and predicted that thyroid cancer might become the fourth most prevalent cancer in 
$2030 .^{3}$ Thyroid cancer is generally classified into four histological subtypes: papillary, follicular, medullary, and anaplastic, among which papillary subtype accounts for $80 \%$ of all the cases. ${ }^{4}$ Surgery, radiation therapy, and thyroid hormone were available for the treatment of thyroid cancer. ${ }^{5}$ Besides, thyroid cancer is featured with slow growth and a favorable prognosis. ${ }^{6}$ However, about $10 \%$ of cases still exhibited worse clinical outcomes due to aggressive behavior such as distant metastasis, or local invasion. $^{7}$ Therefore, it is of urgency to develop effective biomarkers for improving the diagnosis and prognosis of thyroid cancer patients.

As a major lymphatic factor, vascular endothelial growth factor C (VEGFC) plays a regulatory role in angiogenesis and lymphangiogenesis. ${ }^{8}$ It is involved in the lymphatic metastasis process of multiple cancers, such as colorectal cancer, ${ }^{9}$ cervical cancer, ${ }^{10}$ and thyroid cancer. ${ }^{11}$ It has been also demonstrated that overexpression of VEGFC in thyroid cancer led to poor prognosis. ${ }^{12}$ Thus, it is reasonable to refer that VEGFC was a key gene in tumor development.

Solute carrier organic anion transporter family member 4A1 (SLCO4A1), also referred to as OATP4A1, belongs to the SLCO gene family mediating $\mathrm{Na}^{+}$-independent transmembrane transport of various substrates such as therapeutic drugs, thyroid hormones, bile acids, and toxins. ${ }^{13}$ The altered uptake of these substrates may contribute to the changes in the anticancer drug activity, thereby representing a crucial role in the chemosensitivity of cancer cells and affecting the cancer progression. ${ }^{14}$ For instance, SLCO4A1 would be actively induced by cisplatin in NCIH417 cells and had an impact on lung cancer progression and metastasis. ${ }^{15}$ And the elevated expression of prostaglandin E (2) transporting SLCO4A1 caused a decreased sensitivity to cyclic nucleotides in colorectal carcinoma. ${ }^{16}$ Besides, SLCO4A1 was highly expressed in pancreatic cancer and could be a potential biomarker to target anticancer drugs to pancreatic carcinoma. ${ }^{17}$ However, there are few studies on the expression and function of SLCO4A1 in thyroid cancer.

Based on different bioinformatic databases, we first determined SLCO4A1 as the target gene since it had a similar expression and survival pattern to VEGFC, followed by analyzing its expression pattern and prognostic value in thyroid cancer. Then, we identified the coexpressed genes of SLCO4A1 using the LinkedOmics database and performed enrichment analysis to evaluate the biological functions and pathways of SLCO4A1 and its co-expressed genes. Finally, the value of SLCO4A1 in immune therapy was explored.

\section{Materials and Methods}

\section{Acquisition Process of the Target Gene}

Due to the essential role of VEGFC in tumor progression, we determined the target gene taking VEGFC as the starting point. cBioPortal (https://www.cbioportal.org/) and LinkedOmics (http://linkedomics.org/login.php) were used to obtain the genes co-expressed with VEGFC. Through the cBioPortal database, we searched "THCA" and chose "Thyroid Carcinoma (TCGA, Firehose Legacy)" with 516 samples. LinkedOmics is a publicly available portal including multi-omics data from 32 TCGA cancer types and 10 clinical proteomics tumor analysis consortium (CPTAC) cancer cohorts. We screened the co-expressed genes of VEGFC in TCGA_THCA RNA-seq data, and the Pearson correlation test was applied. According to the absolute correlation coefficient, the top 100 co-expressed genes from two databases were respectively selected to screen the consistent co-expressed genes of VEGFC via Venn analysis (http://bioinformatics. psb.ugent.be/webtools/Venn/). Next, an integrated database for genomic and immunogenomic gene set cancer analysis (GSCA) (http://bioinfo.life.hust.edu.cn/GSCA/ $\# /$ ), was employed to examine the survival difference between the high and low expression of the 38 consistent co-expressed genes of VEGFC in thyroid cancer. Then, the expression levels of the 2 genes that were significantly related to worse progression-free survival (PFS) were analyzed. And the prognostic significance of SLCO4A1 was further evaluated through the Kaplan-Meier Plotter website (http://kmplot.com/analysis/index.php? $\mathrm{p}=$ background) by splitting patients with the best cutoff. ${ }^{18}$ Finally, GSCA was used to examine the relationship between drug sensitivity and SLCO4A1 mRNA expression.

\section{Analysis of the SLCO4AI Expression}

The expression level of SLCO4A1 was analyzed using the tumor immune estimation resource (TIMER) (https://cis trome.shinyapps.io/timer/), UALCAN (http://ualcan.path. $\underline{\text { uab.edu/), and human protein atlas (HPA) (https://www. }}$ proteinatlas.org/) databases. TIMER was adopted to initially explore the transcription level of SLCO4A1 in various tumor types. Following this, the mRNA expression of SLCO4A1 in thyroid cancer and normal tissues was evaluated by UALCAN database, which is an interactive web 
resource for analyzing cancer OMICS data. We also assessed the protein localization of SLCO4A1 in human tumor cells as well as the SLCO4A1 protein expression in thyroid cancer and normal tissues through HPA.

Moreover, RNA-seq expression and clinical information based on TCGA were downloaded from the cBioPortal database. We used the TCGA-THCA data composed mainly of papillary thyroid cancer samples to determine the association of SLCO4A1 mRNA expression with clinical parameters such as age, race, and TNM stage. Patients were divided into high and low expression groups by setting median expression as the cutoff. The expression of SLCO4A1 at different clinical stages was generated by UALCAN and gene expression profiling interactive analysis (GEPIA) databases. GEPIA (http://gepia.cancer-pku. cn/detail.php) provides customizable functions such as tumor/normal differential expression analysis, profiling in accordance with cancer types or pathological stages, and patient survival analysis.

\section{Analysis of the Prognostic Significance of SLCO4AI}

We have demonstrated the prognostic role of SLCO4A1 during the acquisition process, we next performed Cox regression analysis to determine independent prognostic factors predicting PFS of thyroid cancer patients. Female was set as a reference level for gender, stage 1 for stage, Asian for race, papillary thyroid cancer-classical for histological type, and bilateral for primary tumor laterality. Moreover, the relationship between SLCO4A1 expression and patient prognosis with restricted clinicopathological characteristics was examined via the Kaplan-Meier Plotter website. Further multivariate analysis of SLCO4A1 expression and PFS in thyroid cancer patients at stage 1 and female thyroid cancer patients was conducted to investigate the independent prognostic value of SLCO4A1 in a specific population. Lastly, the TCGATHCA data were used for receiver operating characteristic (ROC) analysis to verify the important value of SLCO4A1 in distinguishing the survival status of thyroid cancer patients. $P$-value $<0.05$ was set as the cut-off criterion.

\section{Enrichment Analysis of SLCO4AI and Its Co-Expressed Genes}

To elucidate the pathological role of SLCO4A1, the LinkedOmics database was used to screen the genes coexpressed with SLCO4A1. The selection criterion was as follows: cancer cohort, TCGA_THCA; search dataset, RNAseq; search dataset attribute, SLCO4A1; target dataset, RNAseq; statistical method, Pearson Correlation test. Next, the WEB-based gene set analysis toolkit (WebGestalt) (http://www.webgestalt.org/\#) was applied for the enrichment analyses of SLAO4A1 and top 100 coexpressed genes. The advanced parameters were as follows: minimum number of genes for a category, 5; maximum number of genes for a category, 500; number of permutations: 1000. Gene sets with FDR q-value $<0.25$ and $P$-value $<0.05$ were defined as significant pathways. And the positive or negative pathways were determined based on the value of the normalized enrichment score (NES).

\section{Analysis of the Relation Between SLCO4AI and Neutrophil}

First, we used the TIMER database to determine the association of SLCO4A1 with neutrophils, which was validated by TISIDB (http://cis.hku.hk/TISIDB/index.php). TISIDB database is a web portal for tumor and immune system interaction, integrating multiple heterogeneous data types. Then, we assessed how SLCO4A1 expression related to the expression levels of neutrophil markers CEACAM8 and FUT4 using TIMER, verified by the GEPIA database.

\section{Value of SLCO4AI in Immune Therapy}

To investigate the function of SLCO4A1 in immune therapy, the relationship between SLCO4A1 and immune regulators including immunostimulator and immunoinhibitor were examined through the TISIDB database. The threshold was set as $|\mathrm{R}|>0.5, P$-value $<0.05$.

\section{Statistical Analysis}

SPSS 23.0 (SPSS, Inc., Chicago, IL, USA) was utilized to perform all data analyses. The chi-squared test was performed to analyze the relationship between SLCO4A1 levels and clinicopathological factors in thyroid cancer. A $P$-value less than 0.05 was considered statistically significant.

\section{Results}

\section{Identification of SLCO4AI as the Target Gene}

A total of 38 consistent genes co-expressed with VEGFC were identified via Venn analysis (Figure 1A). And these 
A

Genes co-expressed with VEGFC from LinkedOmics

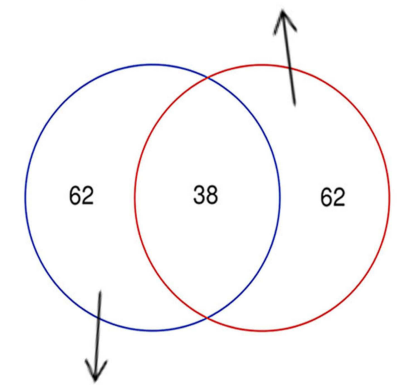

Genes co-expressed with VEGFC from cBioPortal

D

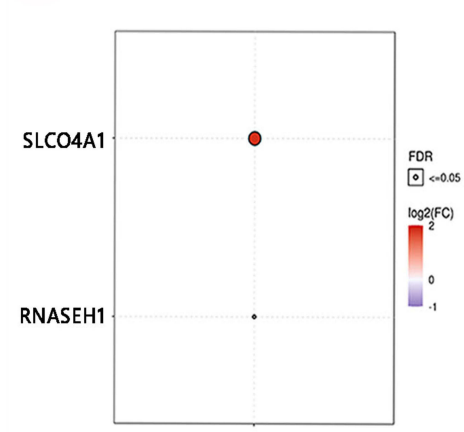

DEGs in thyroid cancer

G

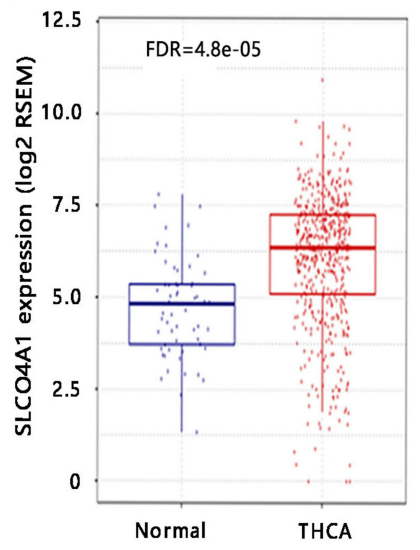

B

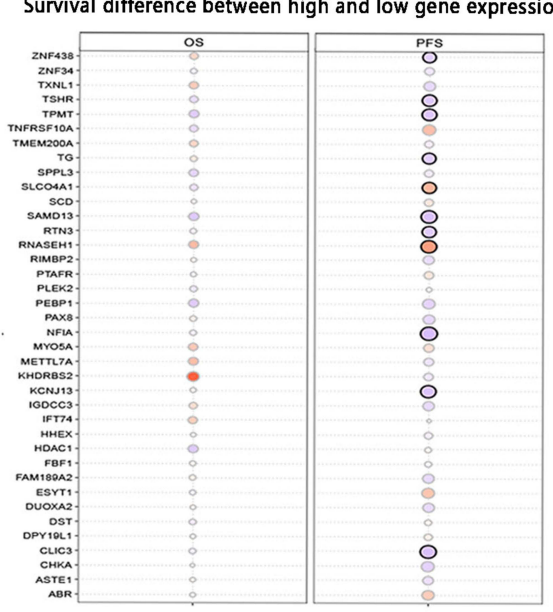

E
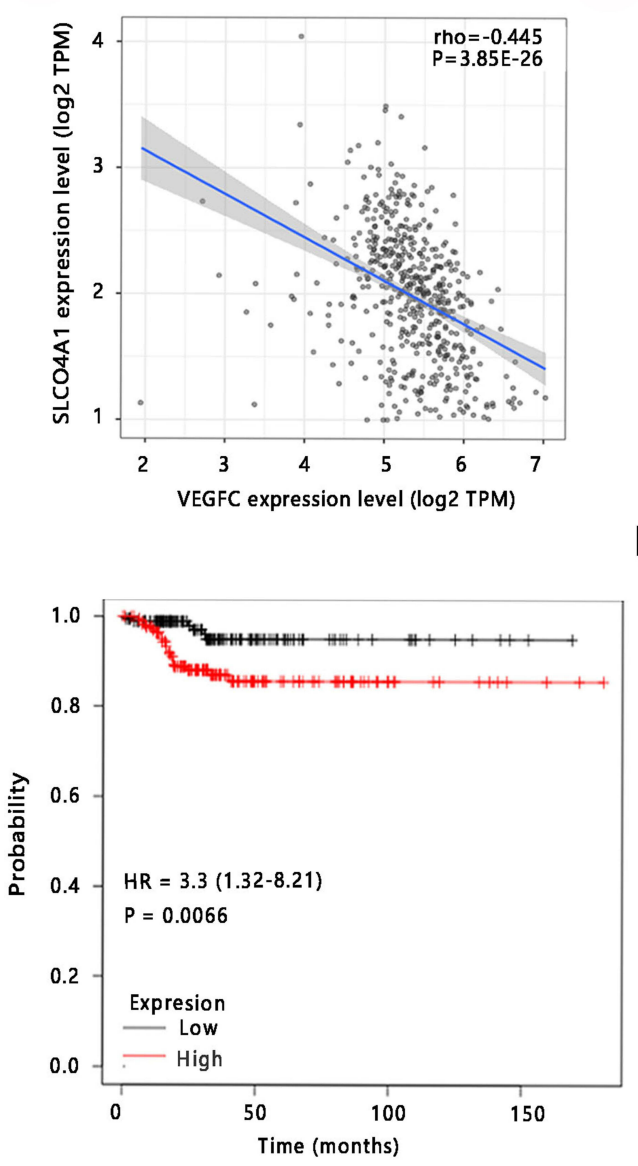

H

$\mathbf{C}_{\text {survival }}$

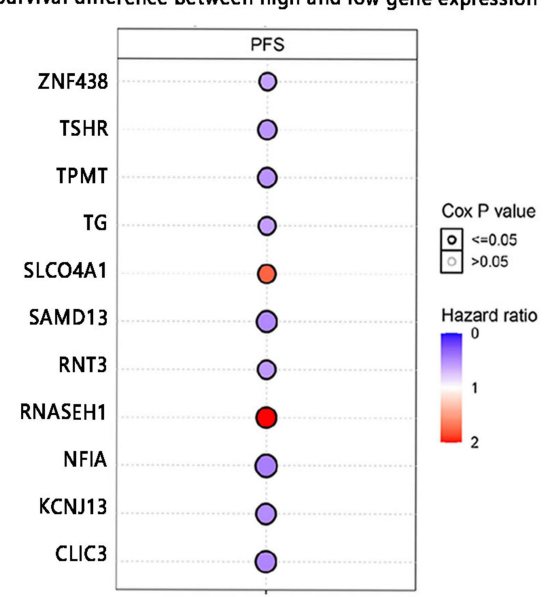

$\mathbf{F}$

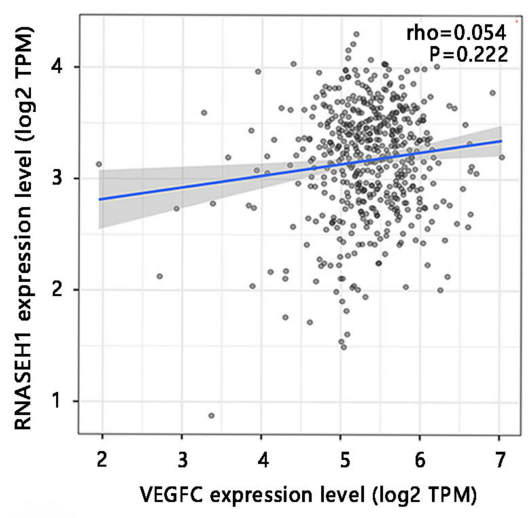

H

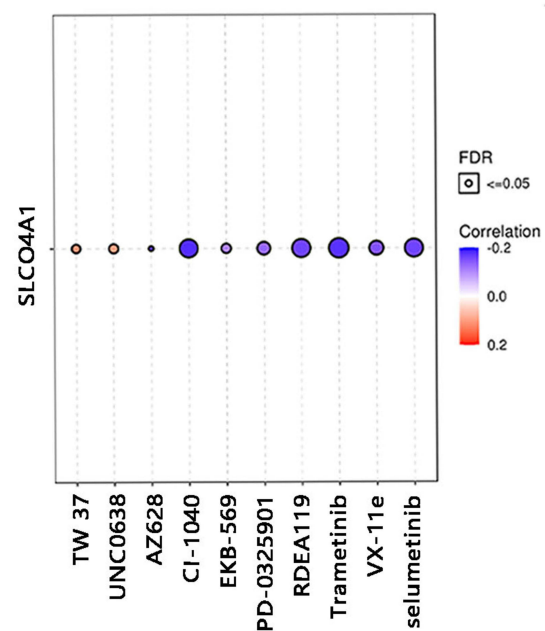

Figure I The acquisition process of the target gene. (A) Screening of the consistent genes that co-expressed with VEGFC. (B) Survival difference between the high and low expression of the 38 genes co-expressed with VEGFC. (C) The significant relationship between II co-expressed genes and PFS. (D) The differential expression analysis of SLCO4AI and RNASEHI. (E) The correlation between SLCO4AI expression and VEGFC expression. (F) The correlation between RNASEHI expression and VEGFC expression. (G) The expression and survival analyses of SLCO4AI in thyroid cancer. (H) The correlation between GDSC drug sensitivity and SLCO4AI mRNA expression.

genes were uploaded into GSCA for survival and expression analysis. No gene was significantly related to OS (all $P>0.05$ ), while 11 genes were remarkably associated with
PFS of thyroid cancer patients $(P<0.05)$ (Figure 1B and $C)$. Notably, high expression levels of SLCO4A1 and RNASEH1 were associated with unfavorable PFS, 
suggesting their clinical significance in thyroid cancer. Thus, the differential expression of SLCO4A1 and RNASEH1 in normal and thyroid cancer tissues was analyzed. The results exhibited that SLCO4A1 mRNA expression was upregulated, while RNASEH1 mRNA expression was downregulated in the thyroid cancer group compared to the normal group (Figure 1D). The above findings indicated that SLCO4A1 was an oncogenic gene. By the Spearman correlation test, VEGFC mRNA expression was closely correlated with that of SLCO4A1 (rho=-0.445) $(P<0.001)$, while its expression was not significantly related to RNASEH1 (Figure $1 \mathrm{E}$ and F). Therefore, SLCO4A1 was selected as the study object.

Following this, the expression pattern and prognostic value of SLCO4A1 were further evaluated. As shown in Figure 1G, SLCO4A1 expression was higher in thyroid cancer tissues in comparison to normal tissues, and its high expression contributed to shorter PFS of thyroid cancer patients. Additionally, SLCO4A1 was negatively related to the drug sensitivity, implying that SLCO4A1 might influence the clinical outcome of thyroid cancer patients by reducing the anti-cancer drug sensitivity (Figure 1H). These results revealed that SLCO4A1 was an oncogenic gene, which might have a significant impact on thyroid cancer development, progression, and treatment. Thus, SLCO4A1 was selected for further analysis.

\section{High SLCO4AI Expression in Thyroid Cancer}

TIMER database was adopted to assess the transcription level of SLCO4A1 in various tumors and normal tissues, presenting that this gene expression was significantly increased in cholangio, colon, pancreatic, rectum, skin, stomach, and thyroid cancer tissues relative to normal tissues, which was decreased in bladder, breast, kidney, liver, and lung cancers (Figure 2A). Then, UALCAN database was used to quantitatively analyze the differential SLCO4A1 mRNA expression in thyroid cancer and normal tissues. The transcription level of SLCO4A1 was elevated in thyroid cancer, with a statistical difference $(P<0.001)$ (Figure 2B). After analyzing the gene expression of SLCO4A1, we next used the HPA database to assess the localization and expression of SLCO4A1 protein. SLCO4A1 protein was expressed in cell junction in human tumor cells, and the immunofluorescence staining result in the A-431 cell line was presented in Figure $2 \mathrm{C}$. In addition, SLCO4A1 intensity was moderate in thyroid cancer and negative in normal tissues, respectively (Figure 2D). Meanwhile, the thyroid cancer tissues showed medium staining, but normal tissues exhibited low staining (Figure 2E). Both of them were incubated with the HPA030669 antibody. These findings suggested an increased expression of SLCO4A1 in thyroid cancer.

\section{Relationship Between SLCO4AI and Clinicopathological Parameters of Thyroid Cancer}

We next investigated the relation between SLCO4A1 mRNA expression and clinicopathological parameters in thyroid cancer. The results showed that mRNA expression of SLCO4A1 was significantly correlated with cancer stage $(\mathrm{P}<0.05)$, while no significant correlation was observed with age, $\mathrm{M}$ stage, $\mathrm{N}$ stage, $\mathrm{T}$ stage, race, gender, histologic type, and primary tumor laterality (Table 1). After a significant relation was found between SLCO4A1 mRNA expression and cancer stage, we used SLCO4A1 mRNA expression as a continuous variable for assessing the association of SLCO4A1 with cancer stage through UALCAN and GEPIA databases. As shown in Figure 3A, patients at stage 4 showed the highest SLCO4A1 level, and patients at stage 2 presented the lowest abundance (all $P<0.05)$. A similar result was observed in the GEPIA database $(P<0.001)$ (Figure 3B). These findings indicated that SLCO4A1 may be closely related to the development of thyroid cancer.

\section{The Association of SLCO4AI with Thyroid Cancer Patient Prognosis}

We subsequently explored the correlation between the expression of SLCO4A1 and the clinical outcome of thyroid cancer patients. During the acquisition process, we have demonstrated that high SLCO4A1 expression led to worse PFS. Therefore, we further assessed the independent prognostic value of SLCO4A1 for PFS of thyroid cancer patients by Cox regression analysis. The univariate analysis results showed that age, stage 3, stage 4, papillary thyroid cancer- tall cell, and SLCO4A1 were significantly related to PFS (all $P<0.05$ ), whereas male, stage 2, African-American, Caucasian, papillary thyroid cancerfollicular, isthmus, left lobe, and right lobe were not. In the multivariate analysis, SLCO4A1 and stage 4 were independent prognostic factors for PFS of papillary thyroid cancer patients (all $P<0.05$ ) (Table 2). Due to the vital value of VEGFC in tumor development, we additionally 


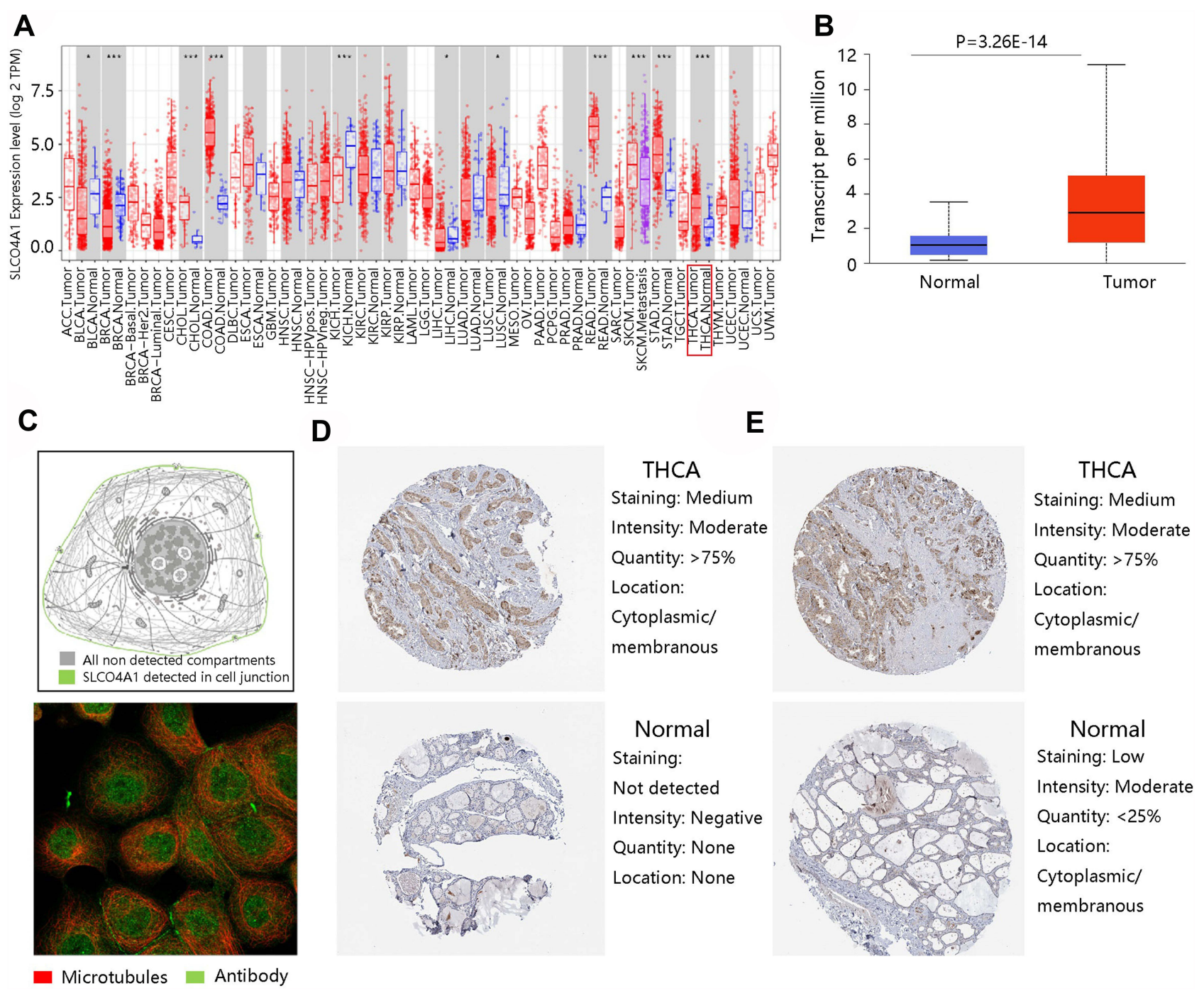

Figure 2 Overexpression of SLCO4AI in thyroid cancer. (A) The gene expression level of SLCO4AI in multiple types of tumor and normal tissues in TIMER. Red box indicated thyroid cancer. (B) SLCO4AI gene expression in normal and thyroid cancer via UALCAN database. (C) The localization of SLCO4AI protein in tumor cells and immunofluorescence staining in the A-43I cell line. (D and E) The protein expression of SLCO4AI in thyroid cancer and normal tissues. $* P<0.05$, ***P $<0.00$ I.

verify the independent prognostic significance of SLCO4A1 by integrating VEGFC into multivariate analysis. SLCO4A1 was still an independent prognostic factor $(P<0.001)$ (Table 3), suggesting that SLCO4A1 had an intimate relation with PFS of papillary thyroid cancer patients.

\section{Increased SLCO4AI Expression is Related to Prognosis in Thyroid Cancer Patients at Stage I and Female Patients} As we observed the association of SLCO4A1 with unfavorable thyroid cancer patient prognosis, we next examined the relation between SLCO4A1 expression and patient prognosis with restricted clinicopathological factors using the Kaplan-Meier Plotter website. As shown in Table 4, SLCO4A1 had no significant impact on OS; however, high SLCO4A1 expression had a close relation with poorer PFS in patients at stage 1 and female subgroups $(P<0.05)$ (Table 4) (Figure 4A and B). Following this, the multivariate analysis of the SLCO4A1 expression and PFS in thyroid cancer patients at stage 1 or female patients was carried out to predict the independent factors. A significant relation was found between SLCO4A1 and PFS of thyroid cancer patients at stage $1(P<0.001)$. In addition, SLCO4A1, stage 3 , and stage 4 were significantly associated with PFS of female thyroid cancer patients (all $P<0.01$ ) (Table 5), indicating that SLCO4A1 functioned as an independent prognostic 
Table I Correlation Between SLCO4AI and Clinical Features in Thyroid Cancer

\begin{tabular}{|c|c|c|c|c|}
\hline \multirow[t]{2}{*}{ Variables } & \multicolumn{2}{|c|}{$\begin{array}{l}\text { SLCO4AI } \\
\text { Expression }\end{array}$} & \multirow[t]{2}{*}{$\chi^{2}$} & \multirow[t]{2}{*}{ P-value } \\
\hline & Low & High & & \\
\hline Age (years) & & & 1.022 & 0.312 \\
\hline$<55$ & 152 & 128 & & \\
\hline$\geq 50$ & 56 & 59 & & \\
\hline M stage & & & 0.275 & 0.600 \\
\hline MO & 118 & 103 & & \\
\hline MI & 3 & 5 & & \\
\hline $\mathrm{N}$ stage & & & 0.125 & 0.724 \\
\hline No & 92 & 81 & & \\
\hline $\mathrm{NI}$ & 97 & 92 & & \\
\hline T stage & & & 3.424 & 0.331 \\
\hline TI & 66 & 47 & & \\
\hline $\mathrm{T} 2$ & 63 & 69 & & \\
\hline T3 & 73 & 63 & & \\
\hline T4 & 5 & 7 & & \\
\hline Cancer stage & & & 8.323 & 0.040 \\
\hline Stage I & 134 & 94 & & \\
\hline Stage 2 & 16 & 19 & & \\
\hline Stage 3 & 41 & 50 & & \\
\hline Stage 4 & 16 & 23 & & \\
\hline Race & & & 0.009 & 0.995 \\
\hline Asian & 21 & 20 & & \\
\hline African-American & 9 & 9 & & \\
\hline Caucasian & 132 & 126 & & \\
\hline Gender & & & 0.065 & 0.799 \\
\hline Female & 150 & 137 & & \\
\hline Male & 58 & 50 & & \\
\hline Histological type & & & 1.892 & 0.388 \\
\hline PTC-classical & 153 & 129 & & \\
\hline PTC-follicular & 39 & 38 & & \\
\hline PTC-tall cell & 12 & 17 & & \\
\hline Primary tumor laterality & & & 1.278 & 0.734 \\
\hline Bilateral & 34 & 34 & & \\
\hline Isthmus & 9 & 9 & & \\
\hline Left lobe & 78 & 60 & & \\
\hline Right lobe & 85 & 82 & & \\
\hline
\end{tabular}

Abbreviation: PTC, papillary thyroid cancer.

factor for PFS of these two specific populations. Thus, the authors referred that SLCO4A1 influenced the clinical outcome of thyroid cancer patients, possibly by affecting the tumor progression, particularly in patients at stage 1 and female patients.

\section{Verification of the Predictive Value of} SLCO4AI by ROC Analysis

To validate the predictive function of SLCO4A1 in distinguishing the survival status in thyroid cancer, the TCGATHCA data were used for ROC analysis. The results exhibited that the area under curve (AUC) of SLCO4A1 expression for OS and PFS was 0.550 (95\% CI: 0.048$1.000 ; P=0.809)$ and 0.626 (95\% CI: $0.536-0.716$; $P=0.010$ ), respectively (Figure 5A and $\mathrm{B}$ ), suggesting that SLCO4A1 might be a useful biomarker to distinguish the PFS status in thyroid cancer, which was consistent with the above findings.

\section{GO and KEGG Analyses of SLCO4AI}

To reveal the underlying mechanism of thyroid cancer, the biological function, and the pathway that SLCO4A1 may be involved in were explored. The genes coexpressed with SLCO4A1 in thyroid cancer were obtained from the LinkedOmics database. The volcano plot showed the SLCO4A1 association results, and the heat map exhibited the co-expressed genes of SLCO4A1 (Figure 6A-C). Then, three categories of GO comprising of biological process (BP), cellular component (CC), and molecular function (MF), together with KEGG enrichment, were performed for SLCO4A1 and top 100 co-expressed genes. For BP terms, SLCO4A1 and its co-expressed genes were mainly enriched in response to stimulus, biological function, and metabolic process. Regarding CC, they were mainly involved in membrane, vesicle, and extracellular space. In terms of MF, they participated in protein binding, ion binding, and hydrolase activity (Figure 6D). KEGG pathway enrichment indicated that SLCO4A1 and its co-expressed genes were involved in the positive regulation of neutrophilmediated immunity $(P<0.05)$ (Figure $6 \mathrm{E})$, revealing that SLCO4A1 might play a critical role in THCA growth and progression via positively regulating neutrophilmediated immunity.

\section{SLCO4AI Expression is Associated with Neutrophil Infiltration Level in Thyroid Cancer}

We have revealed that SLCO4A1 was involved in the positive regulation of the neutrophil-related pathway. Then, the relationship between SLCO4A1 expression and the degree of neutrophil cell infiltration was explored using the TIMER database, verified by the TISIDB. The 
A

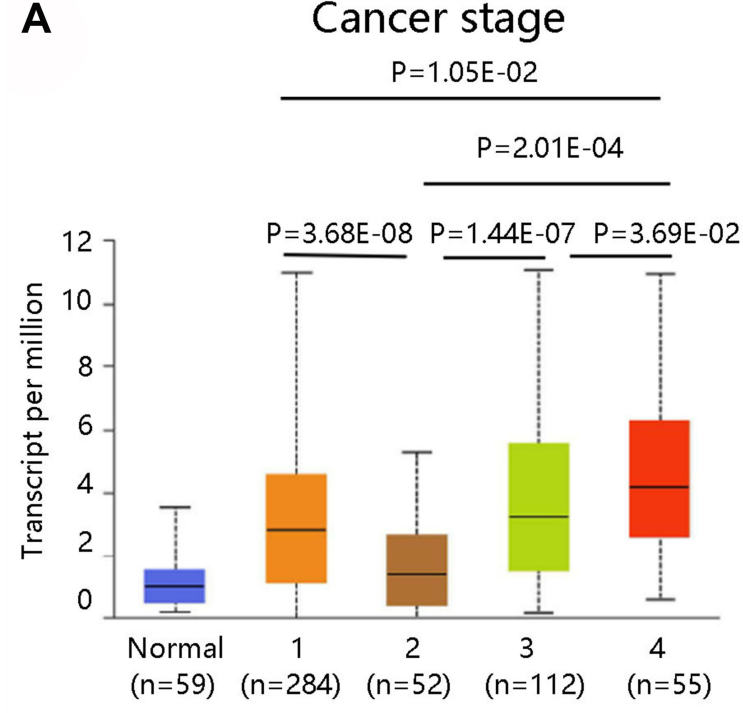

B

Cancer stage

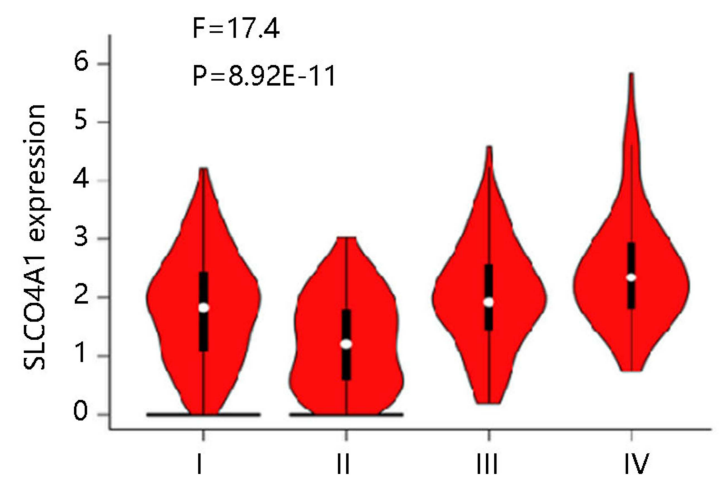

Figure 3 SLCO4AI expression at different stages in thyroid cancer. (A) UALCAN. (B) GEPIA.

results showed that SLCO4A1 expression was positively correlated with neutrophil infiltration level $(P<0.001)$ (Figure 7A and B). After that, the relation between SLCO4A1 expression and neutrophil gene markers in thyroid cancer was explored by TIMER and GEPIA databases. For TIMER, we adjusted these results on basis of tumor purity, demonstrating a significant relation between SLCO4A1 and CEACAM8 $(P<0.001)$ (Figure 7C). However, no significant correlation was found between SLCO4A1 and FUT4 (Figure 7D). Similar correlations were observed of SLCO4A1 expression with the neutrophil markers in GEPIA (Figure 7E and F). Taken together, SLCO4A1 expression had a positive correlation with neutrophil infiltration level, and its gene marker CEACAM8.

\section{The Value of SLCO4AI in Immune Therapy}

As SLCO4A1 was a significant immune infiltration biomarker, we investigated the association of SLCO4A1 with immunostimulators and immunoinhibitors to reveal its value in immune therapy (Figure 8). According to $|\mathrm{R}|$ $>0.5$ and $P$-value $<0.05$, CD274, PDCD1LG2, and IL2RA had a strong correlation with SLCO4A1, indicating

Table 2 Cox Regression Analysis of the SLCO4AI Expression and Progression-Free Survival in Thyroid Cancer Patients

\begin{tabular}{|c|c|c|c|c|c|c|}
\hline \multirow[t]{2}{*}{ Covariates } & \multicolumn{3}{|c|}{ Univariate Analysis } & \multicolumn{3}{|c|}{ Multivariate Analysis } \\
\hline & Hazard Ratio & $95 \% \mathrm{Cl}$ & P-value & Hazard Ratio & $95 \% \mathrm{Cl}$ & P-value \\
\hline Age & 1.026 & $1.005-1.047$ & 0.015 & 1.003 & $0.970-1.038$ & 0.841 \\
\hline Male & 1.452 & $0.745-2.830$ & 0.273 & 1.217 & $0.54 I-2.737$ & 0.634 \\
\hline Stage 2 & 1.210 & $0.350-4.182$ & 0.763 & 1.641 & $0.30 \mathrm{I}-8.952$ & 0.567 \\
\hline Stage 3 & 2.540 & $1.225-5.265$ & 0.012 & 3.032 & $0.980-9.379$ & 0.054 \\
\hline Stage 4 & 3.503 & I.409-8.708 & 0.007 & 5.298 & $1.421-19.753$ & 0.013 \\
\hline African-American & 1.587 & $0.265-9.503$ & 0.613 & 0.851 & $0.132-5.508$ & 0.866 \\
\hline Caucasian & 1.231 & $0.373-4.057$ & 0.733 & 0.753 & $0.216-2.620$ & 0.655 \\
\hline PTC- follicular & 0.749 & $0.288-1.946$ & 0.553 & 0.852 & $0.239-3.046$ & 0.806 \\
\hline PTC- tall cell & 2.528 & $1.040-6.142$ & $0.04 I$ & 1.971 & $0.722-5.379$ & 0.185 \\
\hline Isthmus & 0.350 & $0.044-2.774$ & 0.320 & 0.616 & $0.074-5.109$ & 0.653 \\
\hline Left lobe & 0.549 & $0.233-1.294$ & 0.171 & 0.704 & $0.27 I-1.826$ & 0.470 \\
\hline Right lobe & 0.629 & $0.275-1.438$ & 0.27 I & 0.737 & $0.291-1.868$ & 0.520 \\
\hline SLCO4AI & 1.007 & $1.004-1.009$ & $<0.001$ & 1.008 & $1.005-1.010$ & $<0.001$ \\
\hline
\end{tabular}

Abbreviations: $95 \% \mathrm{Cl}$, confidence interval; PTC, papillary thyroid cancer. 
Table 3 Multivariate Analysis of the SLCO4AI Expression and Progression-Free Survival in Thyroid Cancer Patients

\begin{tabular}{|l|l|l|l|l|}
\hline Covariates & Hazard Ratio & $\mathbf{9 5 \%}$ CI-I & $\mathbf{9 5 \%}$ Cl-u & P-value \\
\hline Age & 1.003 & 0.969 & 1.037 & 0.876 \\
Male & 1.192 & 0.521 & 2.724 & 0.677 \\
Stage 2 & 1.647 & 0.300 & 9.031 & 0.566 \\
Stage 3 & 3.146 & 1.007 & 9.826 & 0.049 \\
Stage 4 & 5.450 & 1.453 & 20.435 & 0.012 \\
African-American & 0.802 & 0.122 & 5.283 & 0.819 \\
Caucasian & 0.706 & 0.202 & 2.465 & 0.585 \\
PTC- follicular & 0.843 & 0.231 & 3.080 & 0.796 \\
PTC- tall cell & 1.939 & 0.709 & 5.304 & 0.197 \\
Isthmus & 0.611 & 0.074 & 5.070 & 0.649 \\
Left lobe & 0.714 & 0.277 & 1.841 & 0.485 \\
Right lobe & 0.733 & 0.289 & 1.854 & 0.511 \\
SLCO4AI & 1.008 & 1.005 & 1.010 & $<0.001$ \\
VEGFC & 1.000 & 0.999 & 1.001 & 0.941 \\
\hline
\end{tabular}

Abbreviations: $95 \% \mathrm{Cl}-\mathrm{I}, 95 \%$ confidence interval lower; $95 \% \mathrm{Cl}-\mathrm{u}, 95 \%$ confidence interval upper; PTC, papillary thyroid cancer.

Table 4 The Correlation Between SLCO4AI Expression and Prognosis in Thyroid Cancer Patients with Different Pathological Factors

\begin{tabular}{|c|c|c|c|c|c|c|}
\hline \multirow[t]{2}{*}{ Clinicopathological Characteristics } & \multicolumn{3}{|c|}{ Overall Survival $(n=502)$} & \multicolumn{3}{|c|}{ Progression Free Survival $(n=353)$} \\
\hline & Number & Hazard Ratio & P-value & Number & Hazard Ratio & P-value \\
\hline \multicolumn{7}{|l|}{ Stage } \\
\hline I & 281 & l & 0.14 & 234 & 5.79 & 0.0092 \\
\hline 2 & 52 & l & 0.2 & 44 & 0.85 & 0.87 \\
\hline 3 & 112 & 0.85 & 0.83 & 75 & 2.17 & 0.28 \\
\hline 4 & 55 & 5.7 & 0.08 & I & l & l \\
\hline \multicolumn{7}{|l|}{ Gender } \\
\hline Female & 367 & 0.69 & 0.57 & 269 & 3.03 & 0.024 \\
\hline Male & 135 & 0.81 & 0.79 & 84 & 1.78 & 0.5 \\
\hline \multicolumn{7}{|l|}{ Race } \\
\hline Caucasian & 332 & & & 227 & 2.4 & 0.064 \\
\hline Asian & 51 & l & 1 & 43 & 0.84 & 0.9 \\
\hline African-American & 27 & I & 0.21 & 12 & l & I \\
\hline
\end{tabular}

that SLCO4A1 might be a potential biomarker for immune therapy in thyroid cancer.

\section{Discussion}

This study determined SLCO4A1 as the target gene by taking VEGFC as the starting point from co-expression, survival, expression, and drug sensitivity analyses. Upregulated SLCO4A1 gene and protein expression levels were found in thyroid cancer, and high SLCO4A1 mRNA expression predicted worse PFS for thyroid cancer patients. Cox regression analysis indicated that SLCO4A1 was a robust independent prognostic factor for PFS of papillary thyroid cancer patients, particularly for those at stage 1 and females. Besides, SLCO4A1 was positively involved in the neutrophil-mediated immunity pathway. Further analyses showed a positive relation between SLCO4A1 and neutrophils and revealed its value in immune therapy.

We initially obtained 38 consistent VEGFC co-expressed genes from cBioPortal and LinkedOmics databases. After the survival and expression analyses, we found that SLCO4A1 was an oncogene. Moreover, a strong correlation was observed between SLCO4A1 and VEGFC. Interestingly, we found that SLCO4A1 had a strong negative association with drug sensitivity, which strengthened its role in the prognosis of thyroid cancer. Veronika et al pointed out that many cytotoxic drugs are substrates for SLCO4A1, and a cancer-specific expression pattern of SLCO4A1 had an 
A

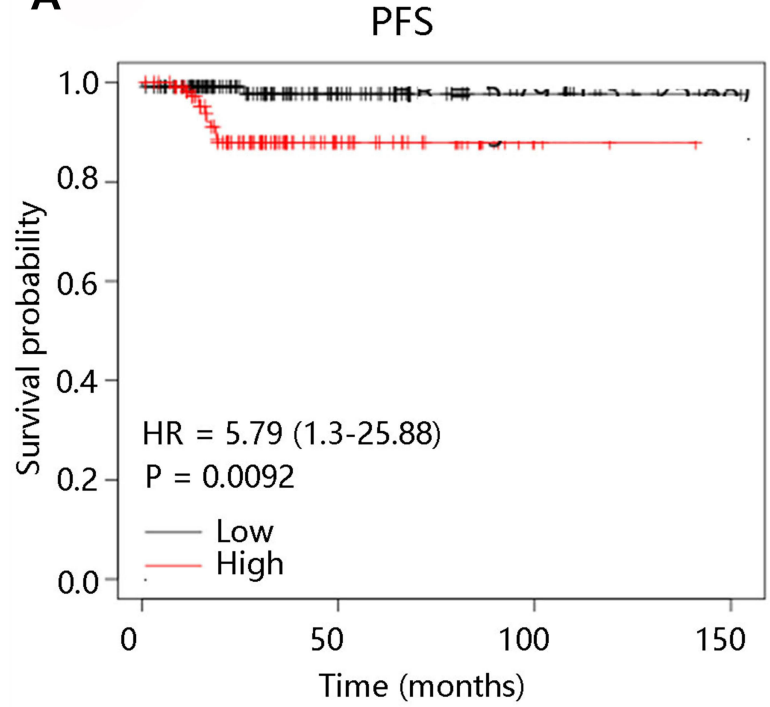

Number at risk

$\begin{array}{llcl}\text { Low } 119 & 33 & 11 & 1 \\ \text { High } 115 & 32 & 3 & 0\end{array}$

B

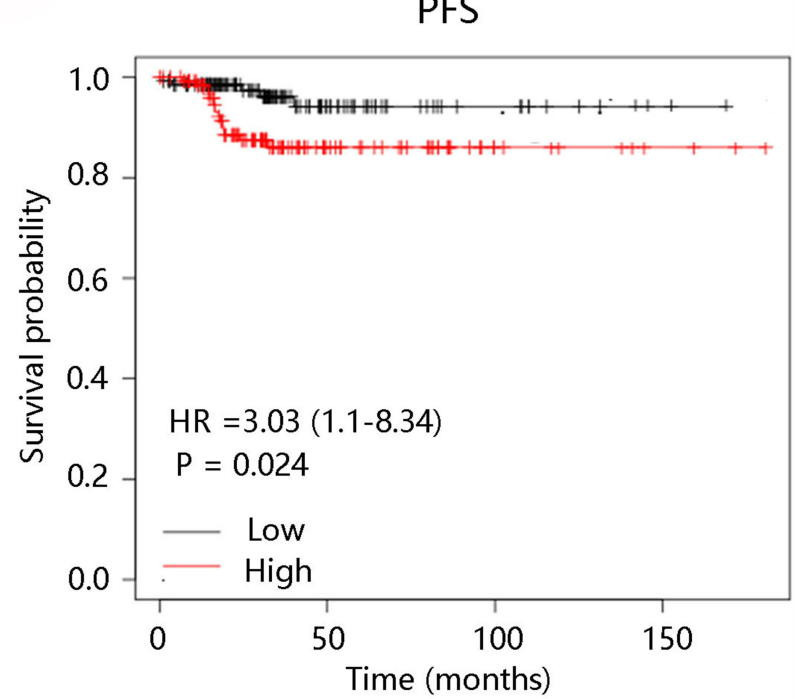

Number at risk

$\begin{array}{lllcl}\text { Low } 135 & 37 & 11 & 2 \\ \text { High } 134 & 35 & 9 & 3\end{array}$

Figure 4 The high SLCO4AI expression predicted poor PFS in thyroid cancer patients at stage I and female patients. (A) Stage I. (B) Female.

impact on the intracellular accumulation of these drugs, and hence the abnormal SLCO4A1 expression would influence the drug sensitivity in cancer cells. ${ }^{14}$ Based on these observations, the authors speculated that high SLCO4A1 expression might affect thyroid cancer patient prognosis by reducing anti-cancer drug sensitivity, indicating an essential impact of SLCO4A1 on thyroid cancer.
Previous studies have demonstrated the aberrant expression of SLCO4A1 in various cancers with multiple results. High expression level of SLCO4A1 was observed in prostate cancer and predicted poor outcomes of the patients. ${ }^{19}$ Consistent with our results, SLCO4A1 also exhibited an upregulation in the pancreatic tumor, while its expression level was decreased in small cell lung

Table 5 Multivariate Analysis of the SLCO4AI Expression and Progression-Free Survival in Thyroid Cancer Patients at Stage I or Female Patients

\begin{tabular}{|c|c|c|c|c|c|c|}
\hline \multirow[t]{2}{*}{ Covariates } & \multicolumn{3}{|l|}{ Stage I } & \multicolumn{3}{|l|}{ Female } \\
\hline & Hazard Ratio & 95\% Cl-I-u & P-value & Hazard Ratio & 95\% Cl-I-u & P-value \\
\hline Age & 0.944 & $0.865-1.029$ & 0.191 & 0.961 & $0.910-1.016$ & 0.159 \\
\hline Male & 0.536 & $0.061-4.668$ & 0.572 & l & I & I \\
\hline African-American & 2.781 & $0.000-\operatorname{lnf}$ & 0.999 & 0.621 & $0.057-6.759$ & 0.696 \\
\hline Caucasian & $336,973.591$ & $0.000-\operatorname{Inf}$ & 0.976 & 0.490 & $0.133-1.805$ & 0.284 \\
\hline SLCO4AI & 1.009 & $1.005-1.014$ & $<0.001$ & 1.008 & $1.005-1.011$ & $<0.001$ \\
\hline VEGFC & 1.000 & $0.998-1.002$ & 0.995 & 1.000 & $0.999-1.002$ & 0.616 \\
\hline Stage 2 & I & I & I & 4.420 & $0.67|-29| 4 \mid$. & 0.122 \\
\hline Stage 3 & I & I & I & 7.209 & I.549-33.545 & 0.012 \\
\hline Stage 4 & I & I & I & 12.293 & $|.723-87.68|$ & 0.012 \\
\hline PTC- follicular & 0.874 & $0.100-7.642$ & 0.903 & 0.325 & $0.039-2.680$ & 0.296 \\
\hline PTC- tall cell & 5.281 & $0.518-53.890$ & 0.160 & 1.492 & $0.445-5.001$ & 0.517 \\
\hline Isthmus & 0.000 & $0.000-\operatorname{Inf}$ & 0.988 & 0.732 & $0.078-6.896$ & 0.785 \\
\hline Left lobe & 0.927 & $0.141-6.089$ & 0.937 & 0.818 & $0.250-2.674$ & 0.740 \\
\hline Right lobe & 0.778 & $0.140-4.311$ & 0.774 & 0.613 & $0.190-1.979$ & 0.414 \\
\hline
\end{tabular}

Abbreviations: 95\% Cl-I, 95\% confidence interval lower; 95\% Cl-u, 95\% confidence interval upper; PTC, papillary thyroid cancer. 
A

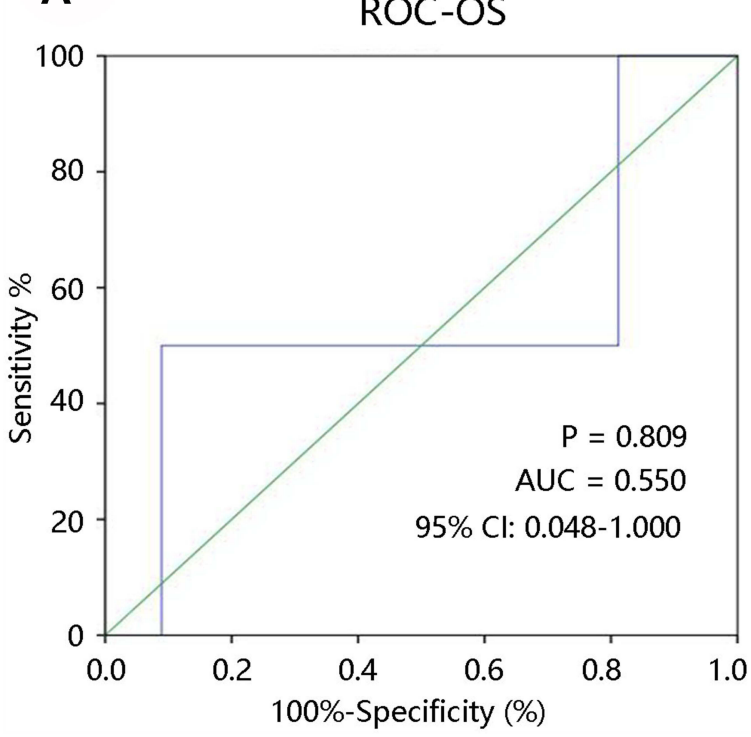

B

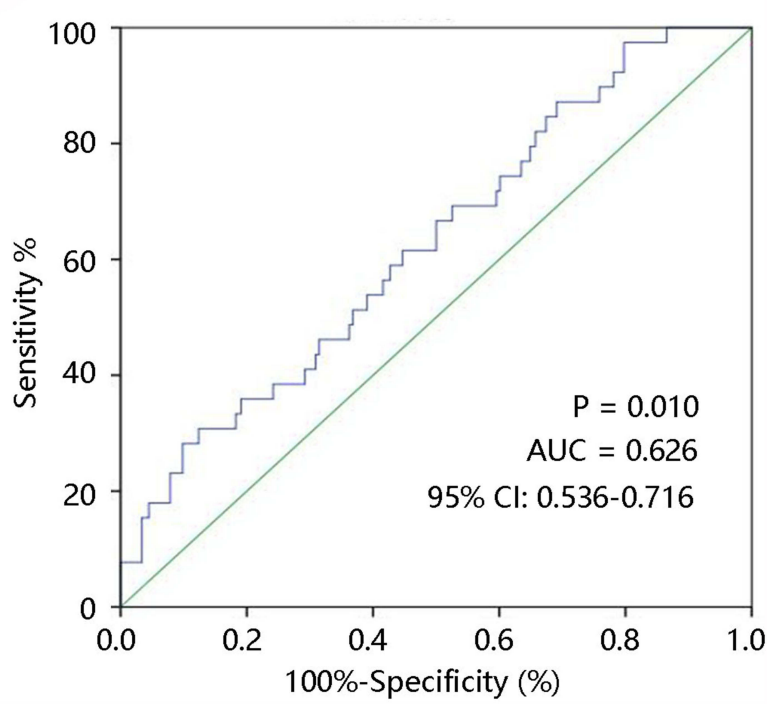

Figure 5 Receiver operating characteristic curves. (A) Overall survival. (B) Progression-free survival.

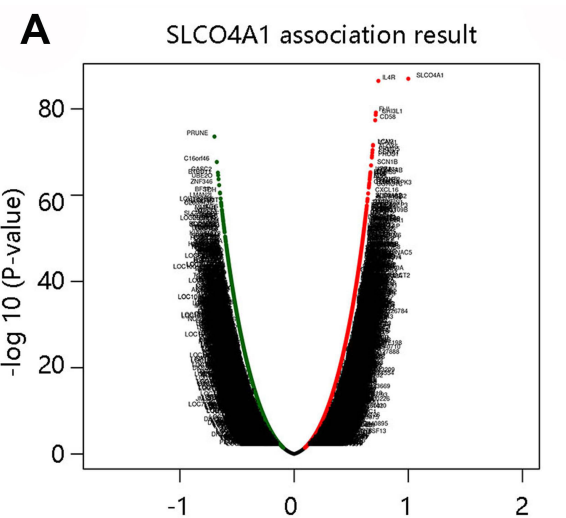

Pearson Correlation Coefficient (Peason test)

D

Biological process

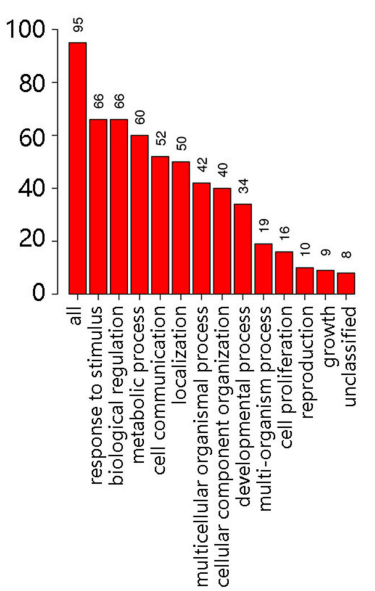

\section{B}

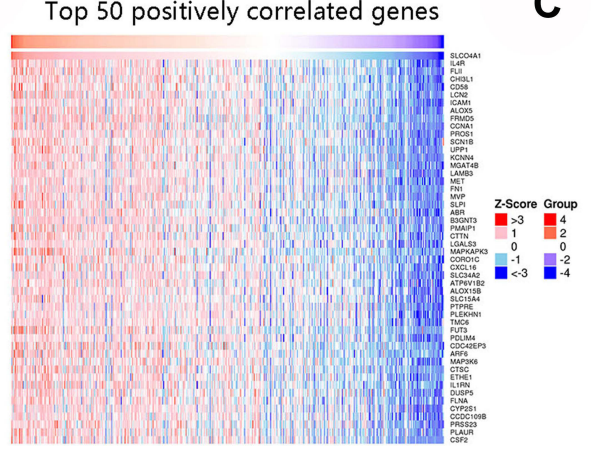

C Top 50 negatively correlated genes

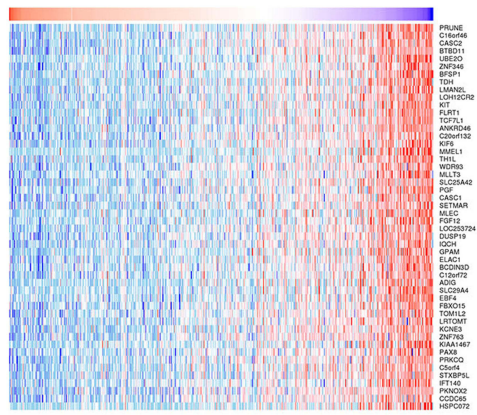

E

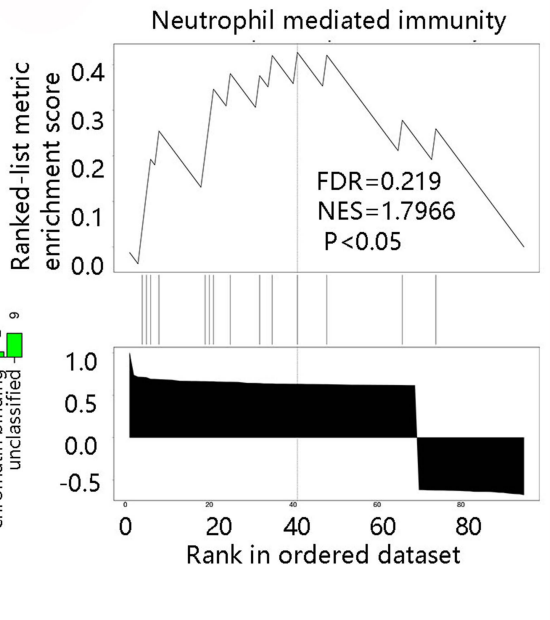

Figure 6 Screening and enrichment analysis of SLCO4AI and its co-expressed genes. (A) The volcano plot of the genes co-expressed with SLCO4AI. (B) The 50 positively correlated genes. (C) The 50 negatively correlated genes. (D) Gene ontology enrichment analysis. (E) KEGG pathway enrichment analysis. 
A

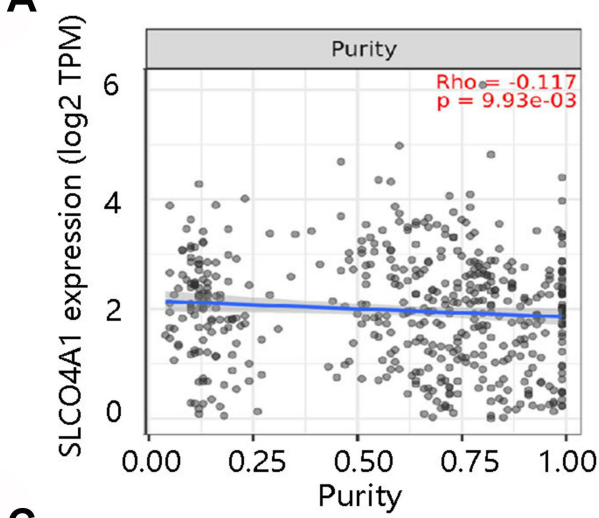

C
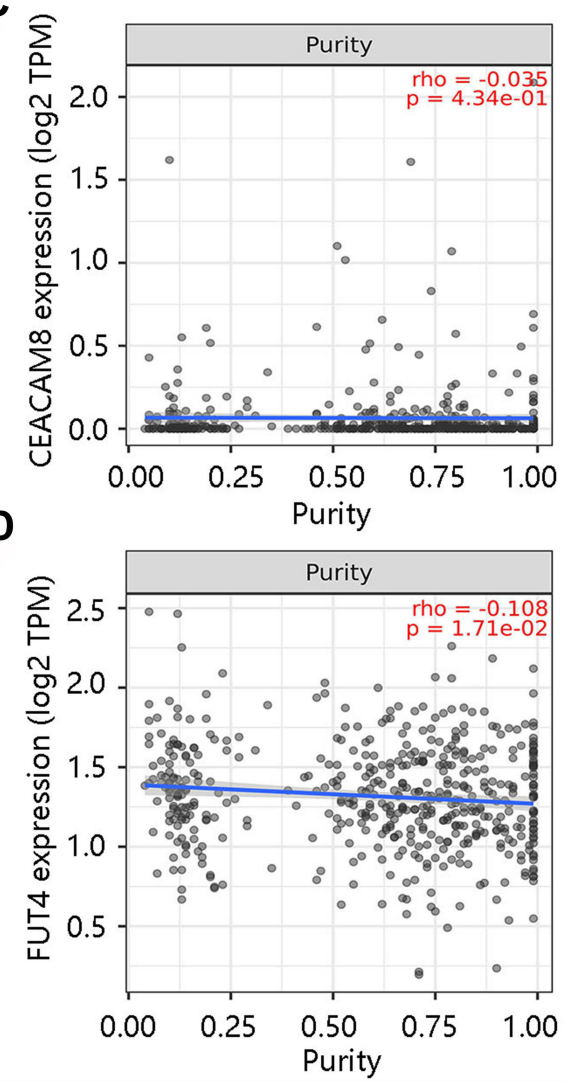
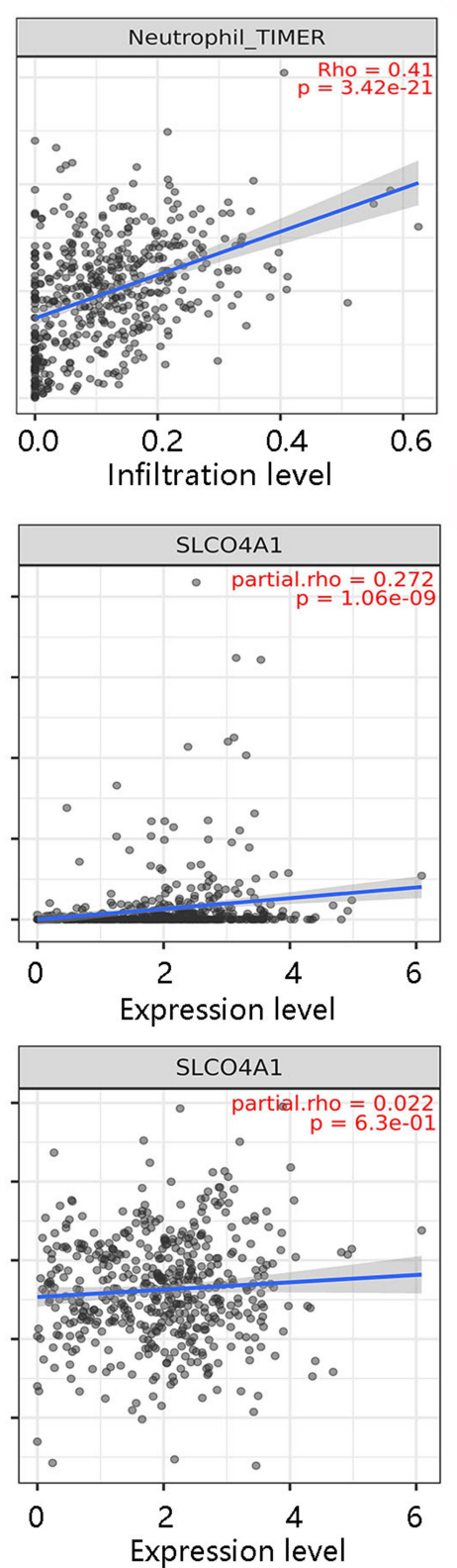

B

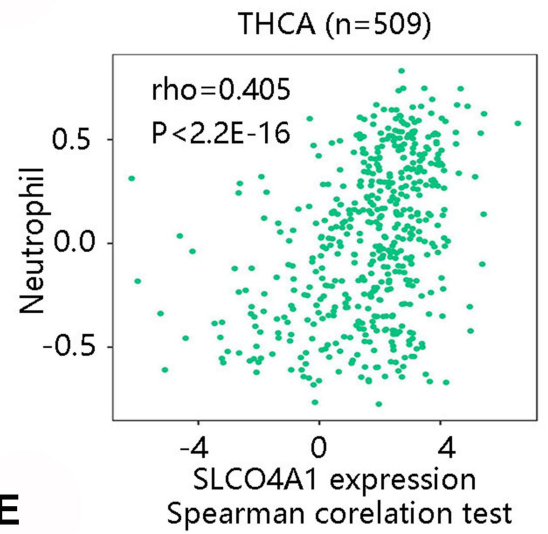

E
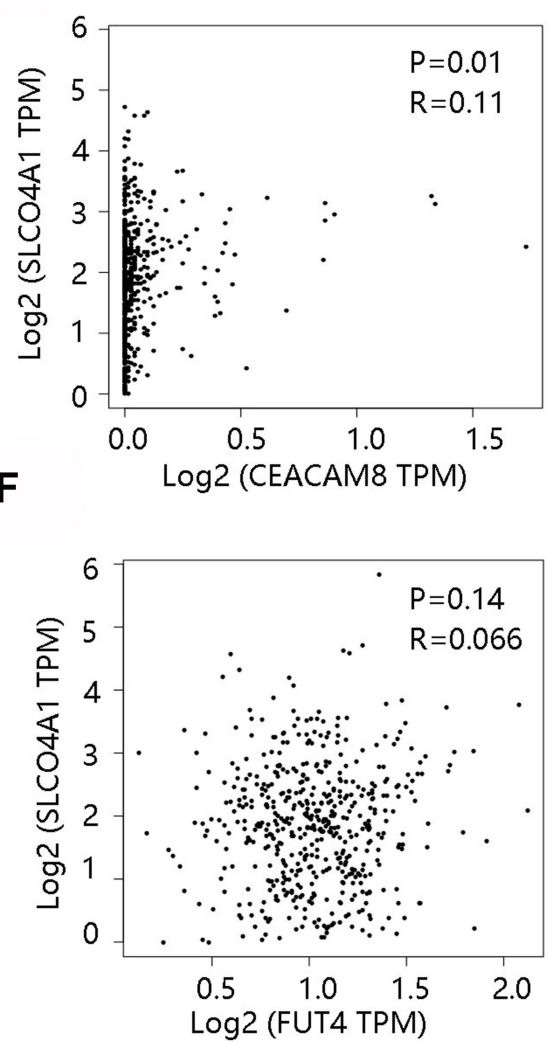

Figure 7 The correlation between SLCO4AI expression and neutrophil infiltration as well as its gene markers. The correlation between SLCO4AI and neutrophil through (A) TIMER, and (B) TISIDB. The correlation between SLCO4AI and neutrophil gene marker CEACAM8 through (C) TIMER, and (E) GEPIA. The correlation between SLCO4AI and neutrophil gene marker FUT4 through (D) TIMER, and (F) GEPIA.

cancer tissue. ${ }^{15,17}$ In this study, SLCO4A1 was highly expressed in thyroid cancer, and its mRNA expression was significantly related to the cancer stage, indicating that SLCO4A1 might be involved in thyroid cancer progression. In addition, our preliminary research found that among the three major histological subtypes (classical, follicular, and tall cell), the classical and tall cell papillary thyroid cancers had elevated SLCO4A1 mRNA expression compared to normal tissues. Interestingly, Yi et al showed that FOXF1 expression was only significantly lower in the classical papillary thyroid cancer than that in adjacent normal tissues. ${ }^{20}$ Then, we explored the prognostic significance of SLCO4A1 in thyroid cancer and we found that SLCO4A1 served as an independent prognostic factor for PFS of papillary thyroid cancer patients. Specifically, SLCO4A1 was significantly related to PFS in thyroid cancer patients at stage 1 or female patients. It has also been reported that SLCO4A1 mediated the cellular uptake 

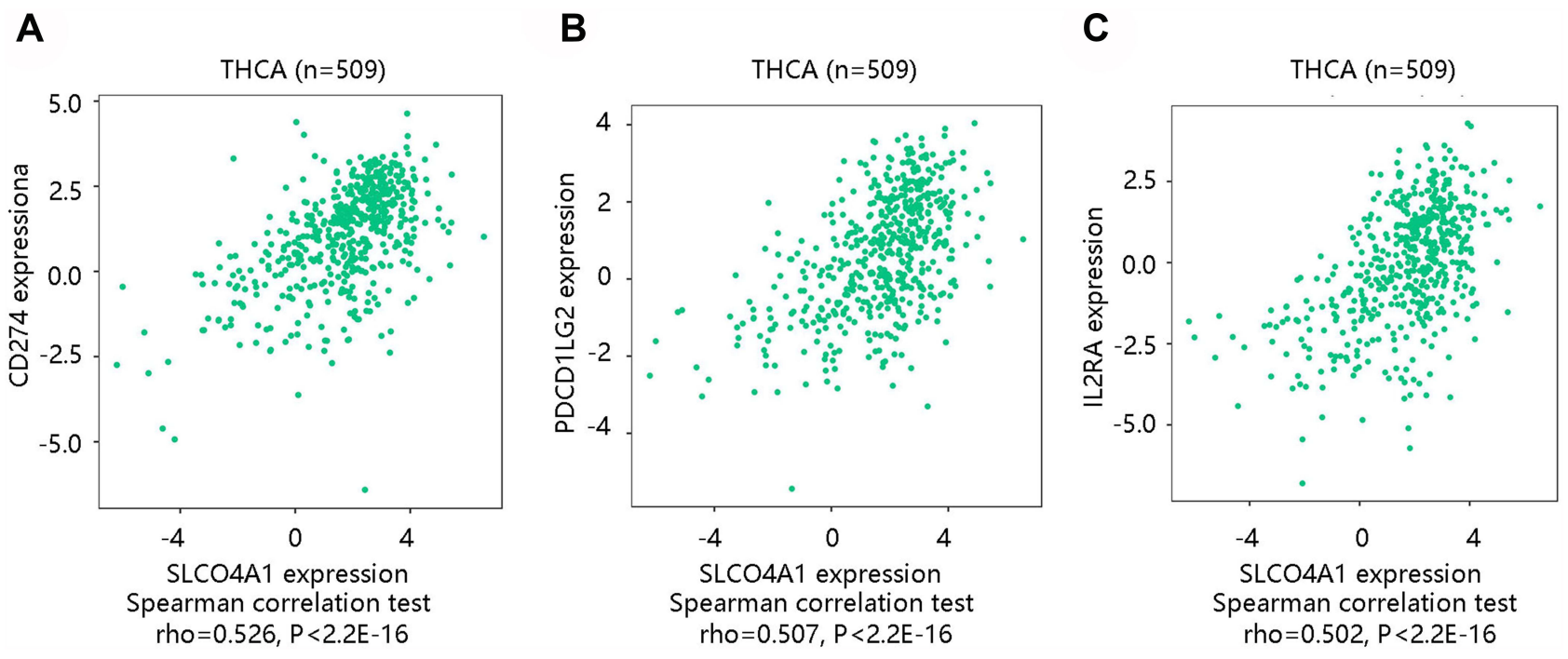

Figure 8 The association of SLCO4AI with immunoregulators in thyroid cancer.

(A) CD274. (B) PDCDILG2. (C) IL2RA.

of many substrates including thyroid hormones, which regulated the progression of cancer through binding the thyroid hormone receptor. ${ }^{21}$ Importantly, thyroid hormone concentration is considered as an independent risk factor for the development and progression of thyroid cancer. ${ }^{22}$ Therefore, the elevated expression of SLCO4A1 might alter the uptake of thyroid hormones, facilitating the progression of thyroid cancer.

To elucidate the underlying mechanism, GO and KEGG analyses of SLCO4A1 and its co-expressed genes in thyroid cancer were performed. BPs were mainly enriched in response to stimulus, biological function, and metabolic process. CCs were mainly enriched in membrane, vesicle, and extracellular space. MFs were mainly enriched in protein binding, ion binding, and hydrolase activity. Besides, SLCO4A1 was positively related to the neutrophilmediated pathway. An additional key finding of the study was that SLCO4A1 had a positive association with neutrophil infiltration and its immune marker CEACAM8. These results suggested that SLCO4A1 was related to tumor growth and metastasis, promoting the progression by positive regulation of the neutrophil-related pathway. High infiltration level of neutrophils contributes to worse prognosis, advanced-stage cancer, and lower PFS, which supported the conclusion of our study. ${ }^{23}$ Finally, we explored the value of SLCO4A1 in immune therapy, and found a strong correlation between SLCO4A1 and immunomodulators including CD274, PDCD1LG2, and IL2RA, making it a possible biomarker for the immune treatment of thyroid cancer.
Although further investigations by in vitro and in vivo experiments were needed to validate the results, our study provided strong theoretical support for the significant value of SLCO4A1 in the diagnosis and prognosis of papillary thyroid cancer.

\section{Conclusions}

In summary, SLCO4A1 may be a valuable biomarker in the papillary thyroid cancer diagnosis and prognosis, and an important target for the treatment of this cancer. Moreover, SLCO4A1 might affect the development of thyroid cancer by positively regulating the neutrophilmediated immunity pathway.

\section{Data Sharing Statement}

The datasets used and/or analyzed during the current study are available from the corresponding authors upon reasonable request.

\section{Ethics Statement}

The Ethics Committee of the Qinghai Provincial People's Hospital agreed to submit the study for review and has waived the need for ethical approval, as the data in this study are from public bioinformatics databases.

\section{Disclosure}

The authors report no competing interests in this work. 


\section{References}

1. Torre LA, Siegel RL, Ward EM, et al. Global cancer incidence and mortality rates and trends-an update. Cancer Epidemiol Biomarkers Prev. 2016;25(1):16-27. doi:10.1158/1055-9965.EPI-15-0578

2. Gao B, Guo L, Luo D, et al. Steroid receptor coactivator-1 interacts with NF- $\mathrm{KB}$ to increase the VEGFC level in human thyroid cancer. Biosci Rep. 2018;38:BSR20180394. doi:10.1042/BSR20180394

3. Rahib L, Smith BD, Aizenberg R, et al. Projecting cancer incidence and deaths to 2030: the unexpected burden of thyroid, liver, and pancreas cancers in the United States. Cancer Res. 2014;74 (11):2913-2921. doi:10.1158/0008-5472.CAN-14-0155

4. Cooper D, Doherty GM, Haugen BR, et al. Revised American Thyroid Association management guidelines for patients with thyroid nodules and differentiated thyroid cancer: the American Thyroid Association (ATA) guidelines taskforce on thyroid nodules and differentiated thyroid cancer. Thyroid. 2009;19(11):1167-1214. doi:10.1089/thy.2009.0110

5. Qi Y, Ji M, Guan H, et al. Shikonin inhibits thyroid cancer cell growth and invasiveness through targeting major signaling pathways. J Clin Endocrinol Metab. 2013;98(12):E1909-E1917. doi:10.1210/jc. $2013-2583$

6. Luster M, Mäder U, Giovanella L, et al. European perspective on the 2015 American Thyroid Association Management guidelines for adult patients with thyroid nodules and differentiated thyroid cancer. Proceedings of an interactive international symposium. Thyroid. 2018;28:722-728. doi:10.1089/thy.2017.0352

7. Shoup M, Stojadinovic A, Nissan A. Prognostic indicators of outcomes in patients with distant metastases from differentiated thyroid carcinoma. J Am Coll Surg. 2003;197(2):191-197. doi:10.1016/ S1072-7515(03)00332-6

8. Mushimiyimana I, Tomas Bosch V, Niskanen H, et al.Genomic landscapes of non-coding RNAs regulating VEGFA and VEGFC expression in endothelial cells. Mol Cell Biol. 2021;41(7):MCB-00594.

9. Tacconi C, Correale C, Gandelli A, et al.Vascular endothelial growth factor $\mathrm{C}$ disrupts the endothelial lymphatic barrier to promote colorectal cancer invasion. Gastroenterology. 2015;148(7):1438-1451.

10. Demei M, XiangXin L, YongPing X, et al. Vascular endothelial growth factor $\mathrm{C}$ expression is closely correlated with lymph node recurrence and poor prognosis in patients with early stage cervical cancer. J Int Med Res. 2013;41(5):1541-1549. doi:10.1177/ 0300060513493038

11. Razy N, Wan F, Win TT. Expression of vascular endothelial growth factor and its receptors in thyroid nodular hyperplasia and papillary thyroid carcinoma: a tertiary health care centre based study. APJCP. 2019;20(1):277.
12. Yu X-M, Lo C-Y, Chan W-F, Lam K-Y, Leung P, Luk JM. Increased expression of vascular endothelial growth factor $\mathrm{C}$ in papillary thyroid carcinoma correlates with cervical lymph node metastases. Clin Cancer Res. 2005;11(22):8063-8069. doi:10.1158/1078-0432.CCR05-0646

13. Stieger B, Hagenbuch B. Organic anion-transporting polypeptides. Curr Top Membr. 2014;73(73):205-232.

14. Buxhofer-Ausch V, Secky L, Wlcek K, et al. Tumor-specific expression of organic anion-transporting polypeptides: transporters as novel targets for cancer therapy. J Drug Deliv. 2013;2013:863539. doi:10.1155/2013/863539

15. Brenner S, Klameth L, Riha J, et al. Specific expression of OATPs in primary small cell lung cancer (SCLC) cells as novel biomarkers for diagnosis and therapy. Cancer Lett. 2014;356(2):517-524.

16. Kleberg K, Jensen GM, Christensen DP. Transporter function and cyclic AMP turnover in normal colonic mucosa from patients with and without colorectal neoplasia. BMC Gastroenterol. 2012;12(1):78. doi:10.1186/1471-230X-12-78

17. Hays A, Apte U, Hagenbuch B. Organic anion transporting polypeptides expressed in pancreatic cancer may serve as potential diagnostic markers and therapeutic targets for early stage adenocarcinomas. Pharm Res. 2013;30(9):2260. doi:10.1007/s11095-012-0962-7

18. Lanczky A, Gyorffy B. Web-based survival analysis tool tailored for medical research (KMplot): development and implementation. J Med Internet Res. 2021;23(7):e27633. doi:10.2196/27633

19. Wright JL, Kwon EM, Ostrander EA, et al. Expression of SLCO transport genes in castration-resistant prostate cancer and impact of genetic variation in SLCO1B3 and SLCO2B1 on prostate cancer outcomes. Cancer Epidemiol Biomark Prev. 2011;20(4):619. doi:10.1158/1055-9965.EPI-10-1023

20. Gu Y, Hu C. Bioinformatic analysis of the prognostic value and potential regulatory network of FOXF1 in papillary thyroid cancer. Biofactors. 2019;45(6):902-911. doi:10.1002/biof.1561

21. Moeller LC, Fuhrer D. Thyroid hormone, thyroid hormone receptors, and cancer: a clinical perspective. Endocr Relat Cancer. 2013;20(2): R19-R29. doi:10.1530/ERC-12-0219

22. He LZ, Zeng T-S, Pu L, et al. Thyroid hormones, autoantibodies, ultrasonography, and clinical parameters for predicting thyroid cancer. Int J Endocrinol. 2016;2016:1-11. doi:10.1155/2016/8215834

23. Granot Z. Neutrophils as a therapeutic target in cancer. Front Immunol. 2019;10:1710. doi:10.3389/fimmu.2019.01710
International Journal of General Medicine

\section{Publish your work in this journal}

The International Journal of General Medicine is an international, peer-reviewed open-access journal that focuses on general and internal medicine, pathogenesis, epidemiology, diagnosis, monitoring and treatment protocols. The journal is characterized by the rapid reporting of reviews, original research and clinical studies across all disease areas. The manuscript management system is completely online and includes a very quick and fair peer-review system, which is all easy to use. Visit http://www.dovepress.com/ testimonials.php to read real quotes from published authors. 